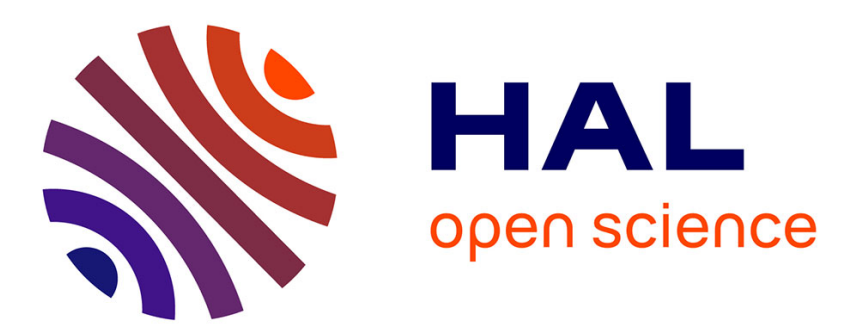

\title{
Evaluation of Telerobotic Shared Control for Efficient Manipulation of Single Cells in Microinjection
}

Jungsik Kim, Dongjune Chang, Hamid Ladjal, David Folio, Antoine Ferreira, Jung Kim

\section{- To cite this version:}

Jungsik Kim, Dongjune Chang, Hamid Ladjal, David Folio, Antoine Ferreira, et al.. Evaluation of Telerobotic Shared Control for Efficient Manipulation of Single Cells in Microinjection. IEEE International Conference on Robotics and Automation (ICRA'2011), May 2011, Shanghai, China. pp.3382-3387, 10.1109/ICRA.2011.5979868 . hal-00624922

\section{HAL Id: hal-00624922 \\ https://hal.science/hal-00624922}

Submitted on 20 Sep 2011

HAL is a multi-disciplinary open access archive for the deposit and dissemination of scientific research documents, whether they are published or not. The documents may come from teaching and research institutions in France or abroad, or from public or private research centers.
L'archive ouverte pluridisciplinaire HAL, est destinée au dépôt et à la diffusion de documents scientifiques de niveau recherche, publiés ou non, émanant des établissements d'enseignement et de recherche français ou étrangers, des laboratoires publics ou privés. 


\title{
Evaluation of Telerobotic Shared Control for Efficient Manipulation of Single Cells in Microinjection
}

\author{
Jungsik Kim, Dongjune Chang, Hamid Ladjal, David Folio, Antoine Ferreira, Member, IEEE, and \\ Jung Kim, Member, IEEE
}

\begin{abstract}
Microinjection is the high delivery efficient method of exogenous materials into cells, and it has been widely used in biomedical research areas such as transgenics and genomics. However, this direct injection task is timeconsuming and laborious followed by low throughput and poor reproducibility. This paper describes telerobotic shared control (TSC) framework for the microinjection with high manipulation efficiencies, in which a micromanipulator is controlled by the shared motion commands of both the human operator (teleoperation) and the autonomous controller. To determine the optimal gains between the teleoperation and automation modules, we propose the quantitative evaluation method using Fitts' and steering law. The results showed that about $60 \%$ weighting on human operator had better performance for both speed and accuracy of task completion, and suggested the some level of automation or human involvement will be important for the microinjection tasks.
\end{abstract}

\section{INTRODUCTION}

$\mathbf{H}$ IGH efficient transfer of foreign materials into cells is important for fundamental research of cell and molecular biology and biomedicine. Several methods have been developed for the successful delivery of exogenous materials into cells. Among them, single cell microinjection is to directly introduce foreign materials, such as DNA, proteins, sperms, and drugs, into individual cells [1]-[3], and it is relatively high efficient delivery method than electrical [4], viral [1], chemical [1], and other transfer methods [5], [6]. Microinjection is used in many different biotechnologies such as cell transfection with DNA in mammalian cells [1], induced pluripotent stem (iPS) cell transfer [7], and in vitro fertilization (IVF) with intracytoplasmic sperm injection (ICSI) [8]. However, although microinjection has high delivery efficiencies, the injection task is time-consuming and labor-intensive work which limit the manipulation of large numbers of single cells [1], [2]. For the elaborate manipulations of delicate and small cells, it demands technical requirements, such as development of micro-motion stages and fabrication of micro pipettes with a fine tip. In addition, the

This research was supported by Basic Science Research Program through the National Research Foundation of Korea(NRF) funded by the Ministry of Education, Science and Technology(2010-0022871).

J.S. Kim and D.J. Chang are with the Department of Mechanical Engineering, Korea Advanced Institute of Science and Technology (KAIST), Daejeon, 305-701, Korea (e-mail: \{js_kim, dongjune.chang $\} @$ kaist.ac.kr)

H. Ladjal, D. Folio, and A. Ferreira are with Institut PRISME, ENSI de Bourges, 88 Boulevard de Lahitolle, 18020 Bourges, France (e-mail: \{hamid.ladjal, david.folio, antoine.ferreira\}@ensi-bourges.fr)

J. Kim is with the Department of Mechanical Engineering, Korea Advanced Institute of Science and Technology (KAIST), Daejeon, 305701, Korea (phone: +82-42-350-3231; fax: +82-42-350-5230; e-mail: jungkim@kaist.ac.kr) great manipulation skills of a human operator are required, who need extensive training to perform the injection tasks, and thus microinjection has low manipulation efficiencies followed by low throughput and poor reproducibility.

The manipulation of micro meter-sized cells is interrupted by limited capability for sensing technologies. The manipulation is performed with insufficient visual information with small field-of-view and narrow depth-of-field, therefore, it is difficult for detection and positioning of cells or glass pipettes. In addition, there is no force/tactile feedback for robot control or haptic interaction. Insensate glass pipettes induce damage itself or of cells.

Several single cell microinjection systems have been proposed to improve the manipulation efficiency. Automated microinjection systems have been developed to remove the human involvement in injection process [9]-[15], where a visual servoing approach is usually used to control the position and force of a micro-manipulator, but it is challenging to create a fully automated manipulation system because microinjection is conducted in diverse and complex conditions such as various cell sizes (from a micro-meter to hundreds of micro-meter) and cell types (floating or attached cells). In addition, they have difficulties in dexterous manipulation of cells with multiple degrees of freedom (DOF) and in target selection (cell nucleus or cytoplasm) in visual servoing. Teleoperated microinejction systems have been developed to provide haptic feedback [16]-[20]. However, most of them have provided force sensing and feedback with a small number of DOF.

As the manipulator control standpoint, this paper presents a telerobotic shared control (TSC) framework for single cell microinjection for high manipulation efficiency. To achieve high throughput and dexterity simultaneously, both automatic and teleoperative functions of system are needed in microinjection. In the shared control approach, a human operator can control manipulator as much as possible while a controller retain cells and glass pipettes in a desired manipulation path or space to satisfy adequate performance. In the remainder of this paper, first, the TSC algorithm is presented to describe the sharing method of the teleoperation- and automationmodule for the microinjection system. In this paper, we focused on providing the quantitative analysis to determine what level of automation (or teleoperation) is better for optimal TSC. Most previous studies for shared control have lack of studies to decide how much the autonomy will be integrated in the teleoperation (or vice versa) for the best performance. Although the shared control has been applied 
to various applications [21]-[24], this work is, to the authors' knowledge, the first application of shared control in cellular micro-manipulation and first providing a evaluation method to determine optimal shared control gains.

\section{METHOD}

Shared control is applied to the microinjection system to compensate the lack of sensing ability in the micro environment, especially for insufficient visual information. First, a planner for the shared control calibrates the coordinates of the micro manipulator and cells, and defines a guidance path to constraint the depth directional motion of the micro manipulator and for the fast approach of the micro manipulator to cells.

\section{A. System Setup}

A biomicromanipulation workstation is designed for single cell microinjection, as shown in Fig. 1(a). In this paper, zebrafish embryos were used as manipulated cells (Fit. 2, [20]). The operator control the master robot (SensAble Technologies, PHANToM Premium 1.0, USA) to input the motion command for the micromanipulator in the slave space. The slave consists in a micromanipulator (PI, F-131, Germany) with injection needle and the other micromanipulator (Sutter, MP225, USA) with a holding glass pipette. The micromanipulator has three translational motion in $X, Y$ and $Z$ direction. The vision system includes a CCD camera (SVS-Vistek, SVS340MUCP, Germany with $640 \times 480$ pixels resolution and maximum of $250 \mathrm{fps}$ ) with microscopic lens (Moritex, MML2-ST65D) to capture images for the cells and the motion of the slave tip.

Fig. 1(b) shows the coordinates of the system considering pure translation. The task space of the master robot is represented by the coordinate frame $\{\mathbf{M}:(x, y, z)\} \in \mathfrak{R}^{3}$, the task space of the micro manipulator is represented by the coordinate frame $\{\mathbf{S}:(X, Y, Z)\} \in \mathfrak{R}^{3}$, and the image space of the fixed camera is defined by the image coordinate frame $\{\mathbf{I}:(u, v)\} \in \mathfrak{R}^{2}$.

\section{B. Planner}

Visual information is a crucial feedback to enable the micro-manipulation tasks. Processing visual data determines the focal plane for depth estimation and the guidance path of the end effector in the image frame. In this study, because the input of manipulator is given in the slave frame $\{\mathbf{S}\}$, the mapping between $\{\mathbf{S}\}$ and the image frame $\{\mathbf{I}\}$ forms a critical component for servoing the slave tip. In order to compute this mapping, a calibration method is developed and implemented. Several calibration methods exist in the literature which are mostly used in macro scale vision applications [25]. Unfortunately, those methods cannot directly be applied to calibrate an optical microscope due to the characteristics of the optical system (e.g., high optical magnifications and very narrow depth of field), and some dedicated methods have been then proposed [26]. In this work, the camera calibration parameters are defined in the focal plane (image space); therefore, the non-sharp tip image,

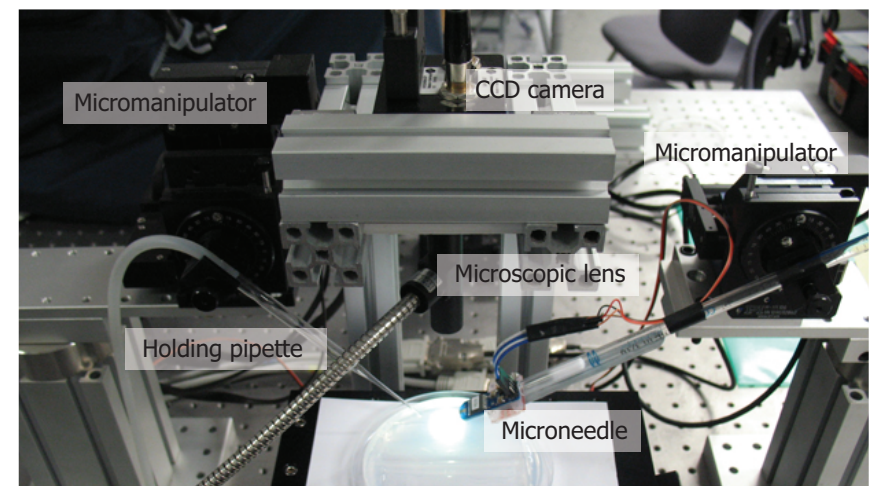

(a)
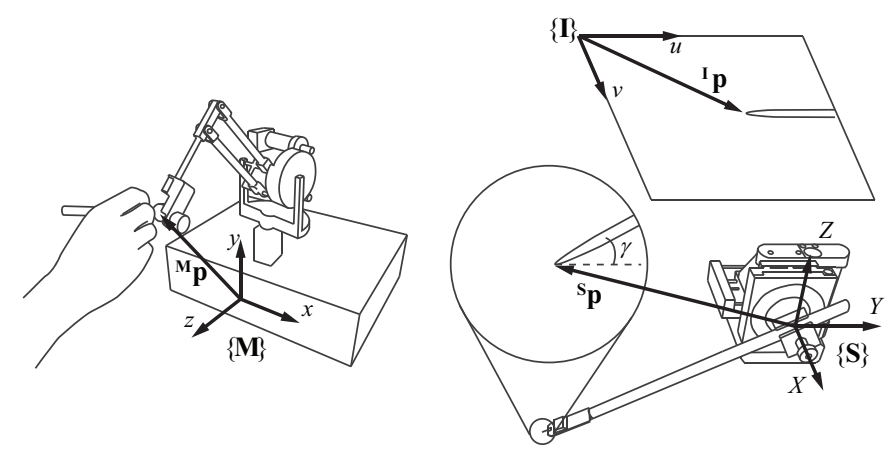

(b)

Fig. 1. (a) Experimental system setup and (b) coordinate frames for master space $\{\mathbf{M}\}$ with haptic device, slave space $\{\mathbf{S}\}$ with micromanipulator, and image space $\{\mathbf{I}\}$. p is position vector and $\gamma$ is the injection angle of a slave tip in the $X$ direction.
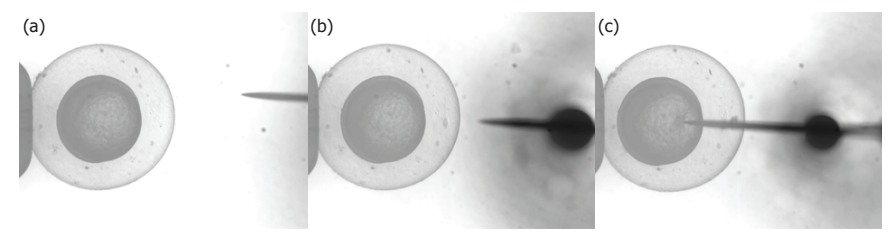

Fig. 2. Cell manipulation process for zebrafish embryo; (a) initial position of the slave tip, (b) starting position of the tip, (c) puncturing chorion (membrane) and approaching to the york.

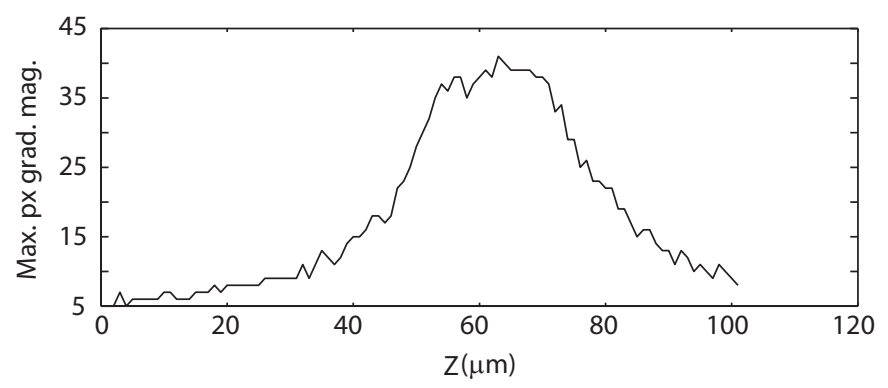

Fig. 3. Maximum pixel gradient magnitudes along the center line of the tip image for different Z-depths. 
due to the depth directional motion of the robot, is ignored. For an orthographic camera projection model, the tip position $\mathbf{S}_{\mathbf{p}}=(X, Y, Z)^{T}$ in the slave frame can be related to its position ${ }^{\mathbf{I}} \mathbf{p}$ in image space as follow:

$$
\mathbf{I} \mathbf{p}=\left(\begin{array}{l}
u \\
v
\end{array}\right)=\left(\begin{array}{l}
Y / S_{u}+u_{0} \\
X / S_{v}+v_{0}
\end{array}\right)
$$

where $S_{u}, S_{v}$ are the pixel dimensions $(\mu \mathrm{m} / \mathrm{px})$, and the initial tip position ${ }^{\mathbf{I}} \mathbf{p}_{0}=\left(u_{0}, v_{0}\right)^{T}$ is measured by the template matching method in the focal plane [20].

When assuming the accurately position-controlled slave manipulator and the orthographic projection model, it is important initially to locate the slave tip in the focal plane. In this paper, the precise placement of the tip along the Zdepth is achieved by the comparison of the pixel gradient magnitudes at the tip-end because the tip is angled and has pure translational motion. The gradient magnitude is at a maximum along the center line in $u$ of the tip image when the tip-end is in the focal plane (Fig. 3).

The position of a cell in the image space is extracted by using Hough transform as the center point of the detected circle, and the focusing of the non-angled holding pipette with a cell was implemented based on the template matching method [9]. Finally, the guidance path is determined as the linear path from the cell position to the injection starting position near the cell membrane, as shown in Fig. 4.

\section{Shared Control}

The overall control architecture of the TSC system is described in Fig 5. The TSC has two levels. The lower level consists of the teleopertion control and the autonomous control based on aritifical potential field (APF). The higher level is the shared controller to integrate both modules. The total input command $\mathbf{S}_{\mathbf{u}}$ for the micromanipulator is defined by the weighted summing of motion commands from the operator and the autonomous controller:

$$
\mathbf{s}_{\mathbf{u}}=K_{h} \mathbf{u}_{h}+K_{c} \mathbf{u}_{c}
$$

where $K_{h}+K_{c}=1$ and $0 \leq K_{h}, K_{c} \leq 1$.

In this paper, the input from the human operator is simply the scaled input command from the master device by

$$
\mathbf{u}_{h}=\alpha_{p}{ }^{\mathbf{M}} \mathbf{p}
$$

APF-based control algorithm [27] is applied for autonomous manipulation module, in which the manipulator is considered as a particle and controlled under a force field by attractive and repulsive potential functions. Attractive potential field is constructed to attract the manipulator to the guidance path, and repulsive potential field is generated around a cell membrane to prevent the micro pipette tip from passing the membrane not in injection direction. The total potential field is defined by the sum of both potential fields, and each potential function is defined as,

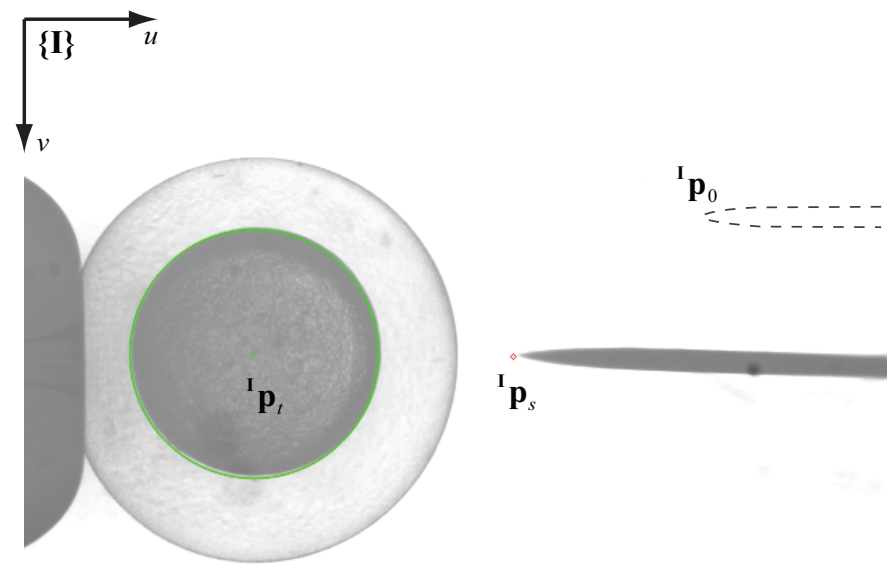

(a)

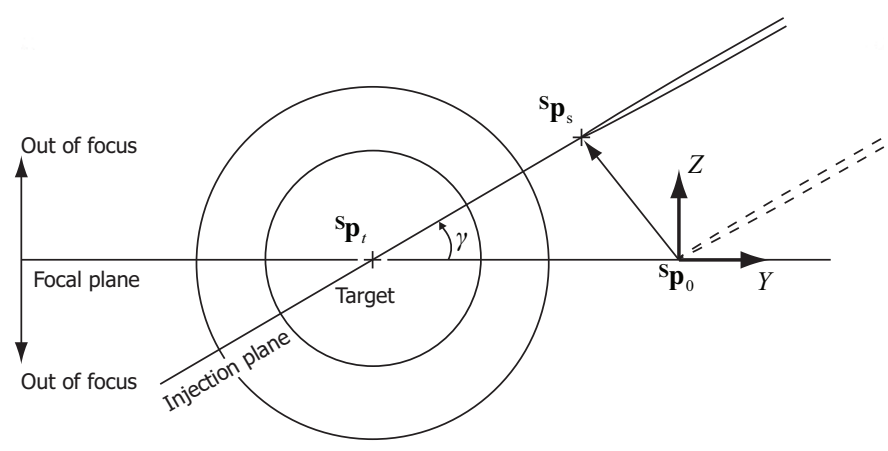

(b)

Fig. 4. (a) Captured image of a cell (zebrafish embryo). ${ }^{\mathbf{I}} \mathbf{p}_{t}$ is the cell position in the york of zebrafish embryo, and green circle represents the edge detection of the york by Hough transform. (b) Path of the slave robot tip in $Y Z$ plane. Subscripts 0 and $s$ represent the initial point and the injection starting point, respectively. Guidance path is defined from ${ }^{\mathbf{S}} \mathbf{p}_{s}$ to ${ }^{\mathbf{S}} \mathbf{p}_{t}$.

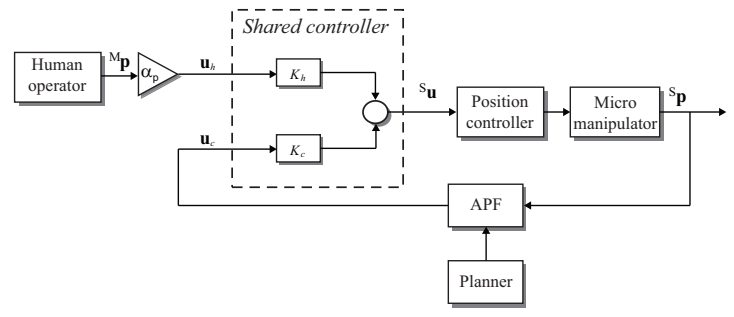

Fig. 5. TSC block diagram.

$$
\begin{aligned}
& U_{\text {total }}(\mathbf{p})=U_{\text {att }}(\mathbf{p})+U_{\text {rep }}(\mathbf{p}) \\
& U_{\text {att }}(\mathbf{p})=\frac{1}{2} K_{a}\left|\mathbf{p}_{d}-\mathbf{p}\right|^{2} \\
& U_{\text {rep }}(\mathbf{p})=\frac{1}{2} K_{r}\left|\mathbf{p}_{o}-\mathbf{p}\right|^{2}, \quad \text { for }\left|\mathbf{p}_{o}-\mathbf{p}\right| \leq r
\end{aligned}
$$

where

$\mathbf{p}_{d}=$ nearest point from the tip to the guidance path

$\mathbf{p}_{o}=$ nearest point from the tip position to the membrane

$K_{a}, K_{r}=$ constant gains

$r=$ radius of influence

Fig. 6 shows 2D potential map in the image space. For the net force $\mathbf{f}_{p}$ under the potential field, finally, the input command from the APF algorithm can be defined as follows, 


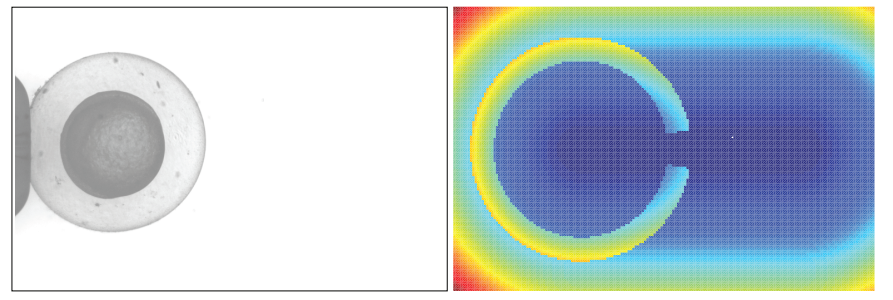

Fig. 6. Total potential field map in the image space. High potential is represented as red color.

$$
\mathbf{u}_{c}=c_{f} \mathbf{f}_{p}=c_{f}\left(-\nabla_{\mathbf{p}} U_{\text {total }}\right)
$$

where $c_{f}$ is compliance constant for modifying input command.

\section{Fitts' and Steering Law}

In the TSC, the input commands from the teleoperation module and the autonomous module to the shared controller have a gain (weighting factor) $K_{h}$ and $K_{c}$, respectively. These gains play a role in deciding which module will be weighted more in the TSC. High human operator weight can lead to that the microinjection system acts as conventional teleoperation system. On the other hand, when more weight is given to automation module, the human operator can lose the control of the micromanipulator.

In this paper, to quantitatively evaluate the effect of weighting level of each module on the TSC and to obtain the optimal gains, we performed 2D pointing and steering tasks based on Fitts' law and steering law. Fitts' and steering law were first developed to quantify the speed and accuracy tradeoff in target-directed movements [28], [29]. They have been applied in various applications, such as human-computer interaction and robotics [30]-[34].

In the Fitts' law, the Movement Time (MT) in seconds to select a target of width $W$ and at distance $A$ is given by,

$$
M T=a+b \log _{2}\left(\frac{A}{W}+c\right)
$$

where $a$ and $b$ are empirical parameters determined by linear regression. $c$ is $0,0.5$ or 1 (we choose as 1 according to [35]). The term $\log _{2}\left(\frac{A}{W}+c\right)$ is referred to Index of Difficulty (ID) in bits which represents the difficulties of the tasks.

In a typical Fitts' law, the trajectory between the initial and final position is ignored. Therefore, if the movement is constrained along a predefined path, steering law well predicts the $M T$ with path length $A$ and width $W$ [29]:

$$
M T=a+b \frac{A}{W} .
$$

Although the shared control between human and robot has been widely studied and various applications for Fitts' law has been presented, the quantitative analysis for choosing ideal weighting in the shared control using Fitts' law has not been studied. In this paper, we present how much weights are ideal for shared control using Fitts' and steering law tests.
TABLE I

TOTAL INDEX OF DIFFICULTY

\begin{tabular}{cccc}
\hline \hline$W(\mathrm{px})$ & $A_{s}(\mathrm{px})$ & $A_{f}(\mathrm{px})$ & $I D_{t}$ \\
\hline 40 & 50 & 90 & 2.95 \\
30 & 50 & 95 & 3.73 \\
40 & 100 & 80 & 4.08 \\
20 & 50 & 80 & 4.82 \\
40 & 200 & 70 & 6.46 \\
30 & 150 & 80 & 6.87 \\
20 & 100 & 70 & 7.17 \\
10 & 50 & 40 & 7.32 \\
30 & 180 & 90 & 8.00 \\
30 & 200 & 90 & 8.67 \\
20 & 150 & 90 & 9.96 \\
20 & 180 & 80 & 11.32 \\
10 & 90 & 90 & 12.32 \\
10 & 100 & 70 & 13.00 \\
10 & 120 & 50 & 14.58 \\
\hline \hline
\end{tabular}

In the proposed TSC for microinjection, a micro pipette guiding task to a cell along the guidance path with the depth directional motion compensation can be seen as steering law task, and a target selection from the guidance path can be Fitts' law task. We propose the model combining Fitts' law and steering law, in which we hypothesized that total index of difficulty $I D_{t}$ is obtained by

$$
\begin{aligned}
& M T=a+b I D_{t} \\
& I D_{t}=I D_{f}+I D_{s}
\end{aligned}
$$

where $I D_{f}$ and $I D_{s}$ are defined in Eq. 6 and Eq. 7, respectively, and $I D_{t}$ is dimensionless number.

\section{EXPERIMENTAL RESULTS}

\section{A. Protocol}

Six subjects (6 males and the age $24.5 \pm 2.3$ years) participated in the experimental task. All subjects were right handed (dominant hand for all subjects) and inexperienced in microinjection and teleoperation system. Fig. 7 shows the task window for the proposed model Eq. 8.

The steering task was performed first for the tunnel (box 1 in Fig. 7). The subjects were asked to guide a white plus sign (the micro pipette tip position) from the right side to the left side of the tunnel without crossing the up or down side of the tunnel. After passing the tunnel, they had to tap inside box 2 by clicking the button on the stylus of the haptic device. The subjects were asked to perform the task as quickly as possible. If the subjects fail to perform the task, the white plus sign changes to the red plus sign, and the trial is restarted with initializing the timer. A completion time was recorded in seconds from the time entering the tunnel to the time tapping box 2, and the number of errors was also recorded.

Four different gains $\left(K_{h}=\{0.2,0.4,0.6,0.8\}\right)$ and fifteen total indices of difficulty (Table. I) were selected. The subjects were not informed about the shared control idea. They performed 4 sets of trials and each set consisted of 60 trials $\left(15 I D_{t} \times 4\right.$ gains $)$ at random order. The first set was training set to practice the task, and the data from other 3 sets were collected. 


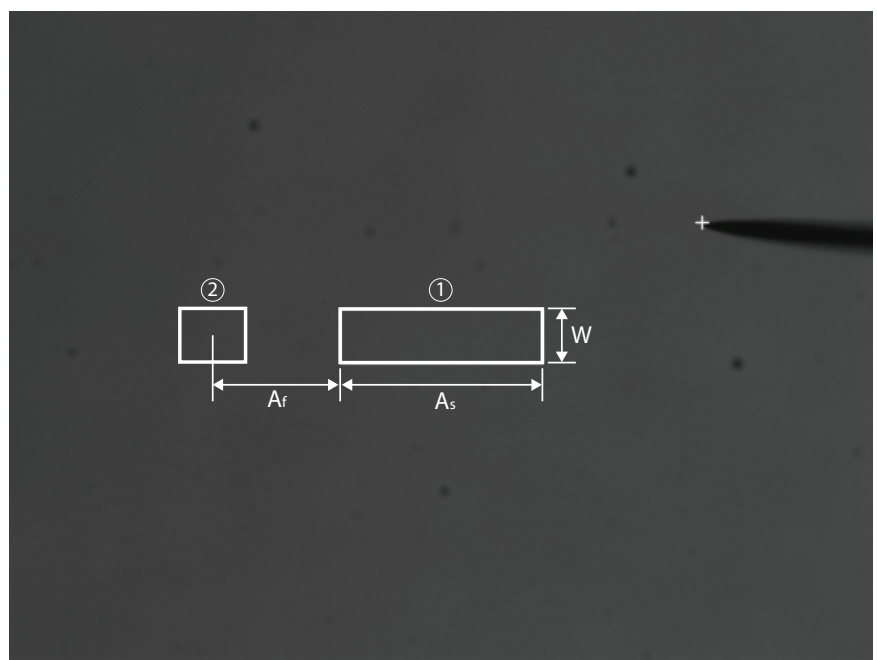

Fig. 7. The Fitts' and steering law task window for the proposed model. A plus sign represents the micro pipette tip position. Two boxes are displayed; box 1 is the tunnel for the steering law task and box 2 is the target for the Fitts' law task. Both tasks have same width but different amplitude.

\section{B. Results}

First, for the performance comparison of different gains, throughput $(T P,[33])$ was calculated by $T P=\frac{1}{n} \sum I D_{t i} / M T_{i}$ ( $n$ is the total number of trials) for each subject. Fig. 8 shows TP as the subjects performance $(\mathrm{S} 1 \sim \mathrm{S} 6)$ and Table. II represents the total TP for all subjects. The subjects showed the better performance at $K_{h}=0.6$ (except S1 and S2; they were at $K_{h}=0.4$ ), and when $K_{h}=0.2$, none of the subjects performed better because of the strong constraints of the movements on the guidance path. For the weak constraints $\left(K_{h}=0.8\right)$, the subjects were easy to stray from the guidance path but the performance is better than $K_{h}=0.2$. That might be because of the learning effect due to the one-dimensional experimental task. Total TP showed a tendency with higher performance between 0.4 and 0.6 of $K_{h}$ although there was no significant difference which can be from the different performances between the subjects.

Fig. 9 shows the total number of errors for all trials. More weighting on the human operator, more errors were presented for all subjects, which is that we expected since, for the weak constrains, the accuracy can be lower than higher constraints.

The experimental results are shown in Table. III and Fig. 10 with the linear regression equations. The proposed model (Eq. 8) showed the $r^{2}$ value of more than 0.766 , for which the model can be used to measure the performance. The model with 0.4 and 0.6 of $K_{h}$ presented better fitting than the other values. This might be from that the skills of the subjects exerted a low influence on the performance of those values. That is, it could be said that the performance of the low skilled human operator can be improved with the TSC.

\section{CONCLUSIONS AND FUTURE WORKS}

In the context of the single cell microinjection system, we proposed the TSC to achieve high throughput and dexterity simultaneously. The TSC can compensate the lack of sensing

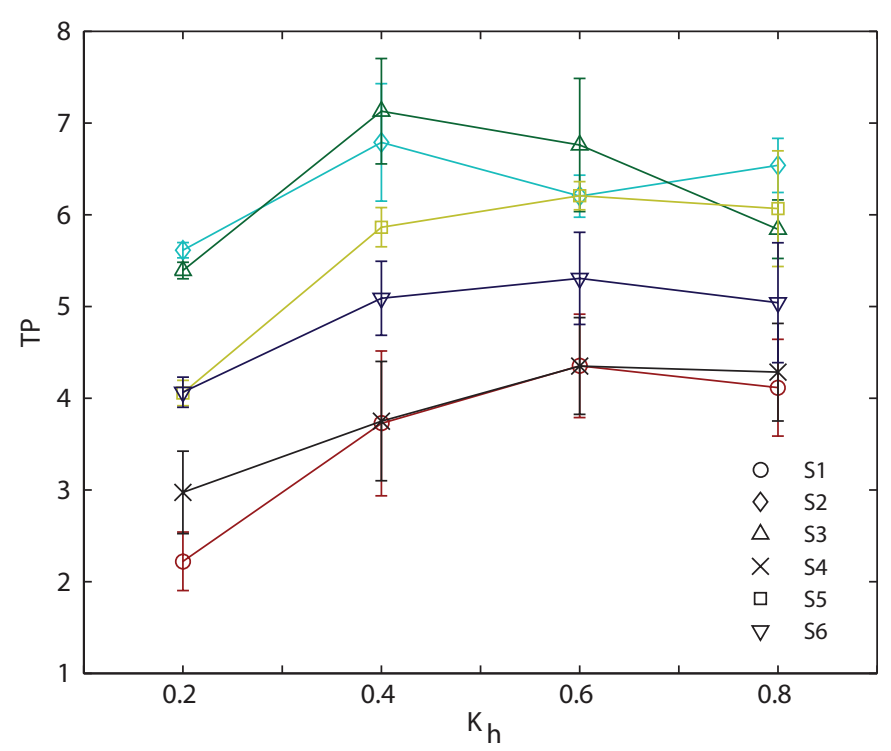

Fig. 8. TP for six subjects from $\mathrm{S} 1$ to $\mathrm{S} 6$.

TABLE II

TOTAL TP FOR TSC GAINS

\begin{tabular}{ccccc}
\hline \hline$K_{h}\left(K_{c}\right)$ & $0.2(0.8)$ & $0.4(0.6)$ & $0.6(0.4)$ & $0.8(0.2)$ \\
\hline $\mathrm{TP}$ & $4.05 \pm 1.32$ & $5.39 \pm 1.47$ & $5.53 \pm 1.02$ & $5.31 \pm 0.99$ \\
\hline \hline
\end{tabular}

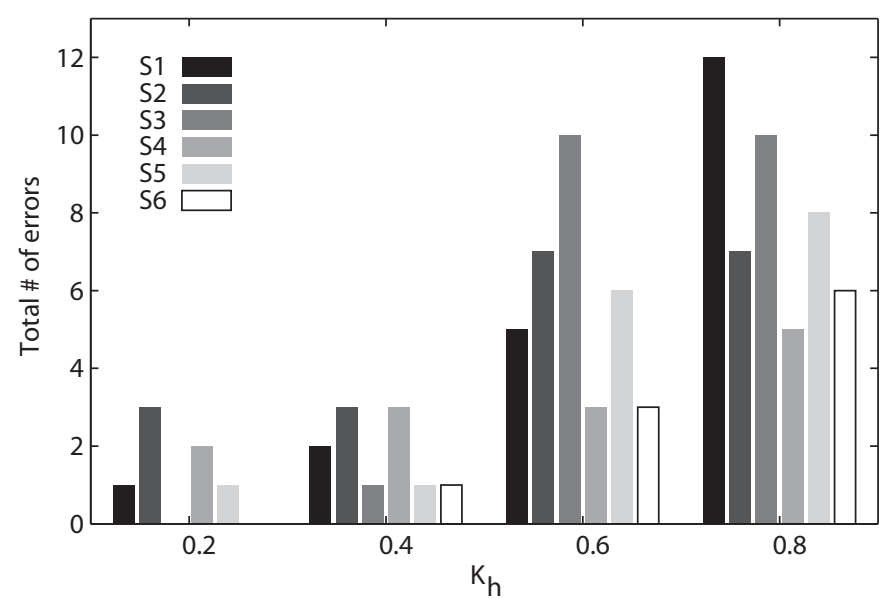

Fig. 9. Total number of errors of the subjects for all trials.

TABLE III

LINEAR REGRESSIONS FOR TSC GAINS

\begin{tabular}{ccc}
\hline \hline$K_{h}\left(K_{c}\right)$ & Model & $r^{2}$ \\
\hline $0.2(0.8)$ & $\mathrm{MT}=1.084+0.139 I D_{t}$ & 0.766 \\
$0.4(0.6)$ & $\mathrm{MT}=0.355+0.165 I D_{t}$ & 0.918 \\
$0.6(0.4)$ & $\mathrm{MT}=0.139+0.187 I D_{t}$ & 0.907 \\
$0.8(0.2)$ & $\mathrm{MT}=0.260+0.190 I D_{t}$ & 0.870 \\
\hline \hline
\end{tabular}




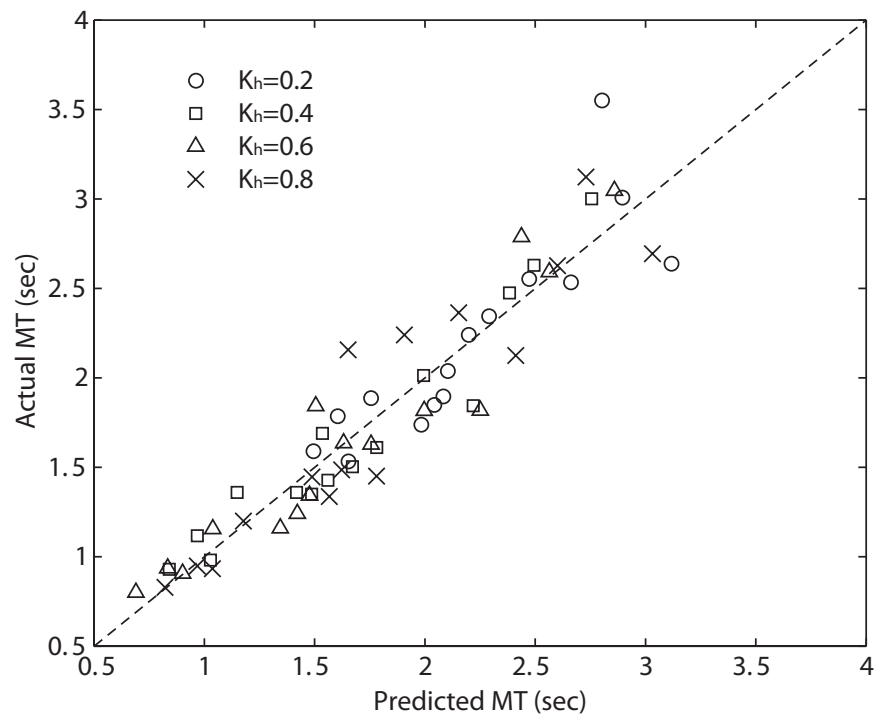

Fig. 10. Relationship between the actual and predicted values (from equations in Table. III) for $K_{h}=0.2$ (open circle), 0.4 (open square), 0.6 (open triangle), and 0.8 (cross). Dashed line represents unit slope for ideal model

ability by providing guidance path for the fast approaching to cells and the assistance with depth directional motions. As an engineering test, the quantitative evaluation of the TSC with the different weighting was performed using Fitts' and steering law. The results showed that the $60 \%$ weighting on human operator had better performance for both speed and accuracy of task completion, and suggested the some level of automation or human involvement will be important for the microinjection tasks.

The design of the optimal control weights on a human operator and a robot is the challenging issue for the shared control. The weighting can be different depending on the application of the shared control idea, for which the proposed method can be used to quantitatively evaluate the performance.

Currently, we are performing the further microinjection experiments with the biological samples (zebrafish embryos [20] and mouse oocytes) to evaluate the TSC system and the clinical evaluation is ongoing. Finally, in our future work, the semi-autonomous microinjection system will be developed with the integration of the TSC and robotic or microfluidic conveying of cells for high throughput and dexterity.

\section{REFERENCES}

[1] D. Luo and W.M. Saltzman, "Synthetic DNA delivery systems," Nature Biotechnology, vol. 18, pp. 33-37, 2000.

[2] D.J. Stephens and R. Pepperkok, "The many ways to cross the plasma membrane," PNAS, vol. 98, no. 8, pp. 4295-4298, 2001.

[3] Y. Zhang and L. C. Yu, "Microinjection as a tool of mechanical delivery," Current Opinion in Biotechnology, vol. 19, pp. 506-510, 2008.

[4] J.E. Bestman, R.C. Ewald, S.L. Chiu, and H.T. Cline, "In vivo singlecell electroporation for transfer of DNA and macromolecules," Nature Protocols, vol. 1, no. 3, pp. 1267-1272, 2006.

[5] U.K. Tirlapur and K. König, "Targeted transfection by femtosecond laser," Nature, vol. 418, pp. 290-291, 2002.
[6] H.J. Kim, J.F. Greenleaf, R.R. Kinnick, J.T. Bronk, and M.E. Bolander, "Ultrasound-Mediated Transfection of Mammalian Cells," Human Gene Theraphy, vol. 7, pp. 1339-1346, 1996.

[7] X.Y. Zhao, Z. Lv, W. Li, F. Zeng, and Q. Zhou, "Production of mice using iPS cells and tetraploid complementation," Nature Protocols, vol. 5, no. 5, pp. 963-971, 2010.

[8] Y. Kimura and R. Yanagimachi, "Intracytoplasmic Sperm Injection in the Mouse," Biol. Reprod., vol. 52, pp. 709-720, 1995.

[9] Y. Sun and B.J. Nelson, "Biological Cell Injection Using an Autonomous MicroRobotic System," Int. J. Robotics Research, vol. 21, no. 10-11, pp. 861-868, Oct.-Nov. 2002.

[10] W.H. Wang, X.Y. Liu, and Y. Sun, "High-Throughput Automated Injection of Individual Biological Cells," IEEE Trans. Autom. Sci. Eng., vol. 6, no. 2, pp. 209-218, 2009.

[11] H. Huang, D. Sun, J.K. Mills, and S.K. Cheng, "Robotic Cell Injection System With Position and Force Control: Toward Automatic Batch Biomanipulation," IEEE Trans. Robot., vol. 25, no. 3, pp. 727-737., 2009.

[12] Y. Xie, D. Sun, C. Liu, S.H. Cheng, and Y.H. Liu, "A Force Control Based Cell Injection Approach in a Bio-Robotics System," in Proc. 2009 IEEE Int. Conf. Robotics and Automation, Kobe, Japan, pp. 34433448 .

[13] M. Lukkari and P. Kallio, "Multi-purpose Impedance-based Measurement System to Automate Microinjection of Adherent Cells," in Proc. 2005 IEEE Int. Symp. Computational Intelligence in Robotics and Automation, Espoo, Finland, pp. 701-706.

[14] L. Mattos, E. Grant, R. Thresher, and K. Kluckman, "Blastocyst Microinjection Automation," IEEE Trans. Inf. Technol. Biomed., vol. 13, no. 5, pp. 822-831, 2009.

[15] Z. Lu, P.C.Y. Chen, J. Nam, R. Ge, and W. Lin, "A micromanipulation system with dynamic force-feedback for automatic batch microinjection,” J. Micromech. Microeng., vol. 17, pp. 314-321, Jan. 2007.

[16] D. Kim, B. Kim, S. Yun, and S. Kwon, "Cellular Force Measuremen for Force Reflected Biomanipulation," in Proc. 2004 IEEE Int. Conf. Robotics and Automation, New Orleans, LA, USA, pp. 2412-2417.

[17] S. Y. Cho and J. H. Shim, "A New Micro Biological Cell Injection System," in Proc. 2004 IEEE/RSJ Int. Conf. Intelligent Robots and Systems, Sendai, Japan, pp. 1642-1647.

[18] M. Ammi, H. Ladjal, and A. Ferreira, "Evaluation of 3D PseudoHaptic Rendering using Vision for Cell Micromanipulation," in Proc. 2006 IEEE/RSJ Int. Conf. Intelligent Robots and Systems, Beijing, China, pp. 2115-2120.

[19] A. Pillarisetti, M. Pekarev, A. D. Brooks, and J. P. Desai, "Evaluating the Effect of Force Feedback in Cell Injection," IEEE Trans. Autom. Sci. Eng., vol. 4, no. 3, pp. 322-331, Jul. 2007.

[20] J. Kim, F. Janabi-Sharifi, and J. Kim, "A Haptic Interaction Method Using Visual Information and Physically Based Modeling," IEEE/ASME Trans. Mechatronics, 2010, accepted for publication.

[21] M. Ammi and A. Ferreira, "Involving the Operator in the Control Strategy for Intelligent Telemicromanipulation," in Proc. 2003 IEEE/ASME Int. Conf. Advanced Intelligent Mechatronics, pp. 868-873.

[22] W.B. Griffin, W.R. Provancher, and M.R. Cutkosky, "Feedback Strategies for Telemanipulation with Shared Control of Object Handling Forces," Presence: Teleoperators \& Virtual Environments, vol. 14, no. 6, pp. 720-731, Dec. 2005.

[23] H.K. Kim, S.J. Biggs, D.W. Schloerb, J.M. Carmena, M.A. Lebedev, M.A.L. Nicolelis, and M.A. Srinivasan, "Continuous Shared Control for Stabilizing Reaching and Grasping With Brain-Machine Interfaces," IEEE Trans. Biomed. Eng., vol. 53, no. 6, pp. 1164-1173., 2006.

[24] U. Bertocchi, L. Ascari, C. Stefanini, C. Laschi, and P. Dario, "HumanRobot Shared Control for Robot-Assisted Endoscopy of the Spinal Cord," in Proc. 2006 IEEE/RAS-EMBS Int. Conf. BioRob., pp. 543548.

[25] Z. Zhang, "A flexible new technique for camera calibration," IEEE Trans. Pattern Anal. Mach. Intell., vol. 22, no. 11, pp. 1330-1334, 2000.

[26] M. Ammi, V. Fremont, and A. Ferreira, "Flexible Microscope Calibration using Virtual Pattern for 3-D Telemicromanipulation," in Proc. 2005 IEEE Int. Conf. Robotics and Automation, Barcelona, Spain, pp. 3888-3893.

[27] O. Khatib, "Real-time obstacle avoidance for manipulators and mobile robots," Int. J. Robot. Res., vol. 5, no. 1, pp. 90-98, 1986.

[28] P.M. Fitts, "The information capacity of the human motor system in 
controlling the amplitude of movement," J. Experimental Psychology, vol. 47, pp. 381-391, 1954.

[29] J. Accot and S. Zhai, "Beyond Fitts's Law: Models for TrajectoryBased HCI Tasks," in Proc. CHI 1997, ACM Press, pp. 295-302, 1997.

[30] D.J. Cannon, "Experiments With a Target-Threshold Control Theory Model for Deriving Fitts' Law Parameters for Human-Machine Systems," IEEE Trans Syst. Man. Cybern., vol. 24. no. 8, pp. 1089-1098, 1994.

[31] O. Tonet, M. Marinelli, G. Megali, A. Sieber, P. Valdastri, A. Menciassi, and P. Dario, "Control of a Teleoperated Nanomanipulator with Time Delay under Direct Vision Feedback," in Proc. 2007 IEEE Int. Conf. Robotics and Automation, Roma, Italy, pp. 3514-3519.

[32] S. Wall and W. Harwin, "Quantification of the effects of haptic feedback during a motor skills task in a simulated environment," in Proc. Phantom User Research Symposium'00, 2000.

[33] R.W. Soukoreff and I.S. MacKenzie, "Towards a standard for pointing device evaluation, perspectives on 27 years of Fitts law research in HCI," Int. J. Human-Computer Studies, vol. 61, pp. 751-789, 2004.

[34] C. Choi, S. Micera, J. Carpaneto, and J. Kim, "Development and Quantitative Performance Evaluation of a Noninvasive EMG Computer Interface," IEEE Trans. Biomed. Eng., vol. 56, no. 1, pp. 188-191, 2009.

[35] I.S. MacKenzie, "A note on the information-theoretic basis for Fitts' law," J. Motor Behavior, vol. 21, pp. 323-330, 1989. 